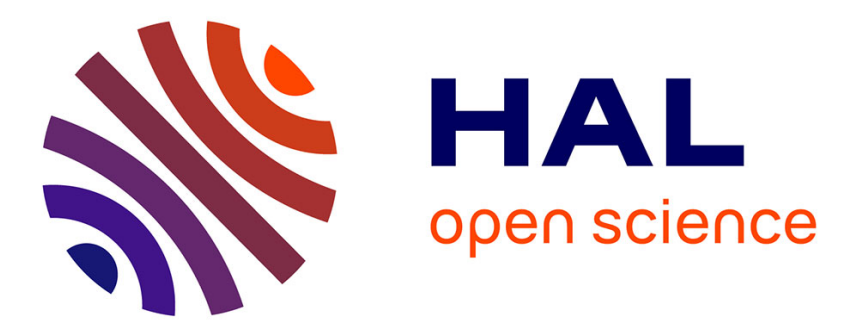

\title{
A constitutive model for semi-crystalline polymer deformation involving lamellar fragmentation
}

Fabrice Detrez, Sabine Cantournet, Roland Seguela

\section{To cite this version:}

Fabrice Detrez, Sabine Cantournet, Roland Seguela. A constitutive model for semi-crystalline polymer deformation involving lamellar fragmentation. Comptes Rendus Mécanique, 2010, 338 (12), pp.681687. 10.1016/j.crme.2010.10.008 . hal-00542497

HAL Id: hal-00542497

https://hal-mines-paristech.archives-ouvertes.fr/hal-00542497

Submitted on 25 May 2020

HAL is a multi-disciplinary open access archive for the deposit and dissemination of scientific research documents, whether they are published or not. The documents may come from teaching and research institutions in France or abroad, or from public or private research centers.
L'archive ouverte pluridisciplinaire HAL, est destinée au dépôt et à la diffusion de documents scientifiques de niveau recherche, publiés ou non, émanant des établissements d'enseignement et de recherche français ou étrangers, des laboratoires publics ou privés. 


\title{
A constitutive model for semi-crystalline polymer deformation involving lamellar fragmentation
}

\section{Une loi de comportement pour les polymères semi-cristallins se déformant par fragmentation}

\author{
Fabrice Detrez $^{\mathrm{a}, \mathrm{b}, *}$, Sabine Cantournet ${ }^{\mathrm{b}}$, Roland Séguéla ${ }^{\mathrm{a}}$ \\ ${ }^{a}$ Univ Lille Nord de France, unité matériaux et transformations, UMR 8207, 59650 Villeneuve d'Ascq, France \\ ${ }^{b}$ Centres des matériaux, UMR 7633, MINES ParisTech, BP 87, 91003 Evry cedex, France
}

\section{Keywords:}

Material engineering

Semi-crystalline polymer

Fragmentation

Damage

Plasticity

Visco-elasticity

Polybutene

Mots-clés:

Génie des matériaux

Polymère semi-crystallin

Fragmentation

Dommage

Plasticité

Visco-élasticité

Polybutène

\section{A B S T R A C T}

The mechanism of lamellar fragmentation in the semi-crystalline polymers with spherulitic structure, is observed at the beginning of plastic flow. It causes significant damage. This elementary mechanism is considered here as a result of plastic deformation coupled with damage, in the framework of generalized standard materials. The simplicity and the efficiency of the proposed approach come from the fact that the semi-crystalline polymers are considered as a macromolecular network bridled by intra-lamellar cohesive forces. Tensile tests and relaxation tests demonstrate the usefulness of a damage-plasticity coupled model.

\section{R É S U M É}

Le mécanisme de fragmentation lamellaire, observé lors de la déformation des polymères semi-cristallins à structure sphérolitique, apparaît et endommage considérablement la microstructure, dès le début de l'écoulement plastique. Dans cette Note, consacrée à la modélisation du comportement de ces matériaux, le mécanisme élémentaire de fragmentation est pris en compte à l'aide d'un couplage plasticité/endommagement. Les développements présentés reposent sur la considération d'un réseau macromoléculaire bridé par les forces cohésives intra-lamellaire. Des essais de traction cyclique et de relaxation, sur le polybutène, valident l'intérêt de l'approche proposée.

\section{Introduction}

Different models that have been proposed in the literature to describe the mechanical behavior spherulitic semicrystalline polymers are not suitable for loading/unloading tensile tests. Important coupling between damage and plasticity has not yet been taken into account. The mechanism of the lamellae fragmentation is the key to understand how the plasticity and damage are coupled. In fact, this mechanism has always been overlooked not only in the thermodynamics-based approaches [1] but also in the micromechanical [2,3].

\footnotetext{
* Corresponding author.

E-mail addresses: detrez@crans.org (F. Detrez), sabine.cantournet@mines-paristech.fr (S. Cantournet), roland.seguela@univ-lille1.fr (R. Séguéla).
} 


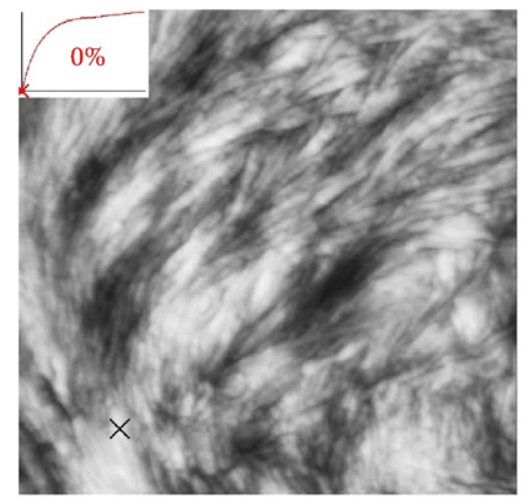

(a) 0
$1.5 \mu \mathrm{m}$

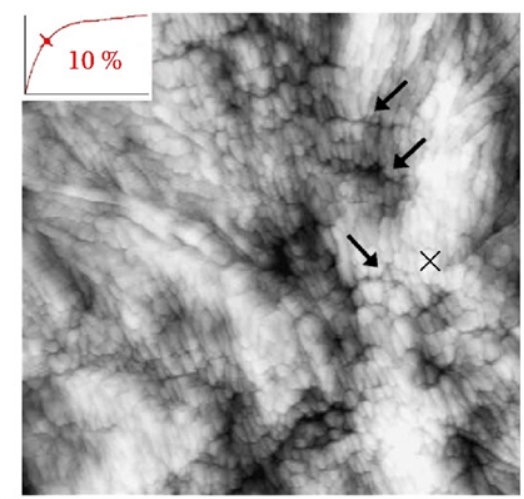

(b) 0
$1.5 \mu \mathrm{m}$

Fig. 1. (a) AFM height image of undeformed $\mathrm{PB}\left(\Delta l / l_{0}=0 \%\right.$ ); (b) AFM height image of deformed $\mathrm{PB} \Delta l / l_{0}=10 \%\left(Z_{\text {range }}=120 \mathrm{~nm}\right)[9]$.

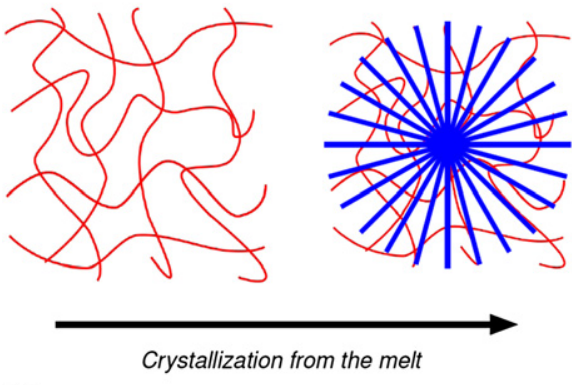

(a) (b)

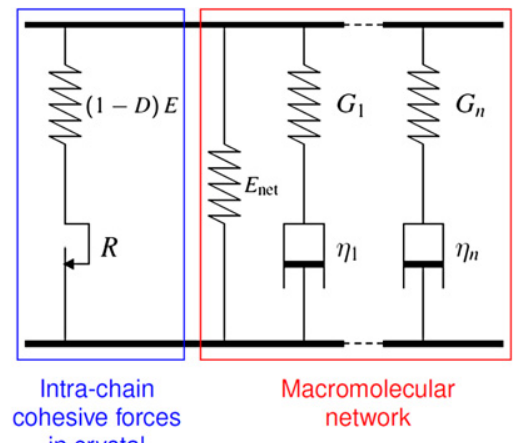

in crystal

Fig. 2. (a) Structural model for spherulitic semi-crystalline polymers; (b) rheological model.

The main purpose of this Note is to derive a new, physically based, constitutive model for describing lamellae fragmentation mechanisms that occur during the deformation process of semi-crystalline polymer. This approach is developed within the framework of the generalized standard materials model [4].

The fragmentation mechanism in semi-crystalline polymer corresponds to the splitting into pieces of the crystalline lamellae. The cracks occur perpendicular to the crystal growth direction. In situ tensile tests were performed under atomic force microscopy (AFM). Fig. 1a shows the undeformed state of the polybutene (PB) and Fig. 1b shows the evolution of polybutene microstructure under deformation. The lamellae (in white Fig. 1) oriented along the tensile direction cracked during the deformation process (black arrows Fig. 1).

\section{Mechanism of lamellae fragmentation in semi-crystalline polymers}

Classically, plasticity in spherulitic semi-crystalline polymers has been theoretically described using crystal slip [2]. The modelling of damage has scarcely been addressed in consideration through cavitation [5]. It is yet important to point out that mechanical damage in semi-crystalline polymers may appear well before necking, i.e. before cavitation can be detected [6].

Furthermore, AFM observations have provided better insight on the lamellae fragmentation process in polypropylene [7] and polyamide 6 [8]. More recently, in situ AFM examination of PB under tensile drawing revealed that lamellae fragmentation takes place at early stages of plastic deformation [9] (Fig. 1). This mechanism of gradual destruction of the spherulitic structure is not only accompanied with permanent strain but also with stiffness loss.

We assume semi-crystalline polymers to consist of a macromolecular network bridled by the intra-lamellar cohesive forces. The rheological model shown in Fig. 2 is used to determine the mechanical behavior of semi-crystalline polymers. Starting from a rubber-like macromolecular network in the melt, the structural model (Fig. 2a) assumes that the spherulitic growth of the crystalline lamellae during solidification leads to a bridling of the entangled chain coils. Indeed it is well known that everything macromolecules of the entangled network run across several crystalline lamellae and amorphous layer so that network and crystal structure are deeply interconnected.

Accordingly, the new model represents the material resistance to deformation with two parallel elements: the first one models the intra-lamellar cohesive forces and the second describes the stretching of entropic network (Fig. 2). The specific free energy could then be split into two terms: 


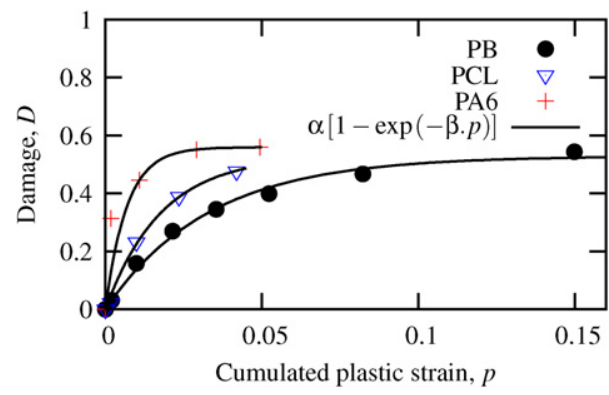

Fig. 3. Damage versus plastic strain for polybutene (PB), polycaprolactone (PCL), polyamide 6 (PA6).

- an energy $\varphi^{c s}$ of a hyperelasto-plastic type with damage variable $D$;

- an energy $\varphi^{\text {net }}$ of visco-hyperelastic type.

The same separation into two terms for the free energy $\varphi$ and for the dissipation potential $\phi$ is applied:

$$
\varphi=\varphi^{c s}+\varphi^{\text {net }} \text { and } \phi=\phi^{c s}+\phi^{\text {net }}
$$

where the superscripts ${ }^{c s}$ and net denote, respectively, the intra-lamellar cohesive forces in crystalline structure and the entropic network.

The contribution of cohesive forces decreases with an increasing deformation, which is due to lamellae fragmentation. This unbridled effect is taken into account by an isotropic damage, variable $D$. Moreover, we have proposed a phenomenological relation coupling damage $D$ and cumulated plastic strain $p$ (see Eq. (13)), based on previous experimental investigations on several semi-crystalline polymers [10] (Fig. 3), given by:

$$
D=\alpha\left(1-e^{-\beta p}\right) \quad \text { where } \alpha \text { and } \beta \text { are material parameters }
$$

This law represents the coupling between the stiffness loss and the permanent strain during the lamellar fragmentation process. The strain rate dependence of semi-crystalline polymer is assumed to be only supported by the dissipation in the macromolecular network.

\section{Cohesive forces in crystalline structure}

\subsection{Kinematics}

For elastic-plastic constitutive description of cohesive forces in the crystalline structure, the deformation gradient tensor $\underset{\sim}{\boldsymbol{F}}=\underset{\sim}{\boldsymbol{F}^{e}} \boldsymbol{F}^{p}$ is decomposed into a plastic and an elastic component, denoted by the superscripts ${ }^{p}$ and ${ }^{e}$. The arbitrary rotation of this decomposition is eliminated by keeping the microstructure orientation constant in the intermediate configuration [11], i.e. the plastic flow is assumed to preserve the microstructure orientation.

\subsection{Thermodynamics potential and state laws}

Let $\mathcal{Z}^{c s}=\{\underset{\sim}{\boldsymbol{C}}, r, D\}$ be a system of state variables. $\underset{\sim}{C}=\underset{\sim}{\boldsymbol{C}^{e T}}$. $_{\sim}^{e}$ represents the elastic right Cauchy-Green strain tensor, $r$ is an isotropic hardening variable and $D$ is a scalar damage variable.

The free energy $\varphi^{c s}$ per unity of mass is defined as:

$$
\varphi^{c s}=(1-D)\left[\varphi^{e}\left({\underset{\sim}{\boldsymbol{c}}}^{e}\right)+\varphi^{p}(r)\right]
$$

Such an approach has been initially developed in small deformation by Saanouni [12]. The same damage factor $(1-D)$ is introduced in the stored free energy via elasticity $\varphi^{e}$ and the stored free energy via hardening $\varphi^{p}$. The elastic free energy is characterized by an isotropic fourth-order elastic modulus $\underset{\approx}{\boldsymbol{A}}$

$$
\varphi^{e}=\frac{1}{2 \rho^{e}}{\underset{\sim}{\boldsymbol{E}}}^{e}: \underset{\sim}{\boldsymbol{\Lambda}}:{\underset{\sim}{\boldsymbol{E}^{e}}}^{e} \text { with }{\underset{\sim}{\boldsymbol{E}}}^{e}=\frac{1}{2}\left(\boldsymbol{C}^{e}-\underset{\sim}{\boldsymbol{I}}\right) \text { and } \rho^{e}=\rho \operatorname{det} \underset{\sim}{\boldsymbol{F}^{e}}
$$

where $\rho$ and $\rho_{e}$ are mass density measured in the current configuration and the intermediate configuration respectively. $\underset{\sim}{\boldsymbol{A}}$ is defined by the Young modulus $(E)$ and Poisson ration $(v)$.

The hardening free energy $\varphi^{p}=\frac{1}{2 \rho^{e}} Q \cdot b \cdot r^{2}$ is fully determined by two constants $(Q, b)$. The state laws are derived from the free energy 


$$
\begin{aligned}
& {\underset{\sim}{\boldsymbol{S}}}^{e}=\rho^{e} \frac{\partial \varphi^{c s}}{\partial \underline{\boldsymbol{C}}^{e}}=\rho^{e}(1-D) \frac{\partial \varphi^{e}}{\partial \underline{\boldsymbol{C}}^{e}}=(1-D) \underset{\approx}{\boldsymbol{\Lambda}}:{\underset{\sim}{\boldsymbol{E}}}^{e} \\
& R=-\rho^{e} \frac{\partial \varphi^{c s}}{\partial r}=-\rho^{e}(1-D) \frac{\partial \varphi^{p}}{\partial r}=-(1-D) b \cdot Q \cdot r \\
& Y=-\rho^{e} \frac{\partial \varphi^{c s}}{\partial D}=\rho^{e}\left(\varphi^{e}\left(\underline{\sim}^{e}\right)+\varphi^{p}(r)\right)
\end{aligned}
$$

The Cauchy stress tensor in crystalline structure $\boldsymbol{\sigma}^{c s}$ is obtained by transport of ${\underset{\sim}{\boldsymbol{S}}}^{e}$ through the current configuration

$$
\stackrel{\sigma}{\sim}^{c s}=\frac{1}{J^{e}} \underset{\sim}{\boldsymbol{F}^{e}} \cdot{\underset{\sim}{\boldsymbol{S}}}^{e} \cdot \boldsymbol{F}^{e^{T}} \text { with } J^{e}=\operatorname{det} \underset{\sim}{\boldsymbol{F}^{e}}
$$

\subsection{Evolution laws}

The approach of Lemaitre $[13,14]$ is adopted as the fragmentation governs both the plasticity and damage. This approach involves a single plastic multiplier $\lambda$ with a single dissipation potential and a single rule of normality. The dual dissipation potential $\phi_{\star}^{c s}$, obtained by Legendre-Fenchel's transform of the dissipation potential $\phi^{c s}$, is written as:

$$
\phi_{\star}^{c s}=\phi_{\star}^{p}(\underset{\sim}{\boldsymbol{\Sigma}}, R ; D)+\phi_{\star}^{D}(Y ; D) \quad \text { with } \underset{\sim}{\boldsymbol{\Sigma}}=\frac{1}{\rho} \boldsymbol{F}^{\boldsymbol{F}^{T}} \cdot \boldsymbol{\sigma}^{c s} \cdot \boldsymbol{\sim}^{e-T}
$$

where $\underset{\sim}{\boldsymbol{\Sigma}}$ is the state force associated with plastic flow. The dissipation dual potentials are chosen as:

$$
\phi_{\star}^{D}(Y ; D)=\frac{\alpha}{\beta} Y \quad \text { and } \quad \phi_{\star}^{p}(\underset{\sim}{\tilde{\Sigma}}, \tilde{R})=f(\underset{\sim}{\tilde{\Sigma}}, \tilde{R})+\frac{\tilde{R}^{2}}{2 Q} \quad \text { with } f(\underset{\sim}{\tilde{\Sigma}}, \tilde{R})=J(\underset{\sim}{\tilde{\Sigma}})-\tilde{R}-R_{0}
$$

where $\underset{\sim}{\tilde{\Sigma}}=\underset{\sim}{\boldsymbol{\Sigma}} /(1-D)$ and $\tilde{R}=R /(1-D)$ are effective state forces. They are defined as the state forces obtained for the same internal state variable $\underset{\sim}{\boldsymbol{c}}{ }^{e}$ and $r$ but with a damage-free material, i.e. $D=0$ [15]. The function $f(\underset{\sim}{\tilde{\Sigma}}, \tilde{R})$ defines the elastic domain. $R_{0}$ is the limit of elasticity and $J(\bullet)$ is the von Mises norm. The normality rule gives:

$$
{\underset{\sim}{\nabla}}^{p}={\underset{\sim}{\dot{\boldsymbol{F}}}}^{p}{\underset{\sim}{\boldsymbol{F}}}^{p-1}=\dot{\lambda} \frac{\partial \phi_{\star}^{c s}}{\partial \underset{\sim}{\boldsymbol{\Sigma}}}=\dot{\lambda} \frac{\partial \phi_{\star}^{p}}{\partial \underset{\sim}{\boldsymbol{\Sigma}}}, \quad \dot{r}=\dot{\lambda} \frac{\partial \phi_{\star}^{c s}}{\partial R}=\dot{\lambda} \frac{\partial \phi_{\star}^{p}}{\partial R}, \quad \dot{D}=\dot{\lambda} \frac{\partial \phi_{\star}^{c s}}{\partial Y}=\dot{\lambda} \frac{\partial \phi_{\star}^{D}}{\partial Y}
$$

The gradient tensor of plastic rate is

$$
{\underset{\sim}{\nabla}}^{p}=\dot{p}{\underset{\sim}{\boldsymbol{n}^{e}}}^{e} \quad \text { with }{\underset{\sim}{\boldsymbol{n}}}^{e}=\frac{\partial \phi^{p}}{\partial \underset{\sim}{\boldsymbol{\Sigma}}}=\frac{3}{2} \frac{\operatorname{dev} \underset{\sim}{\boldsymbol{\Sigma}}}{J(\underset{\sim}{\boldsymbol{\Sigma}})}
$$

because the derivative of accumulated plastic strain is:

$$
\dot{p}=\sqrt{\frac{2}{3} \nabla^{p}: \nabla^{p}}=\frac{\dot{\lambda}}{1-D}
$$

The expression of $\dot{p}$ is obtained using the flow conditions: $f(\tilde{\sim}, \tilde{R})=0$ and $\dot{f}(\tilde{\sim}, \tilde{R})=0$ [16]. It is important to point out that the experimental coupling law (2) is obtained by integrating evolution equation (11-3).

\section{Macromolecular network}

\subsection{Kinematics}

Regarding the visco-elastic constitutive description of the macromolecular network, the deformation gradient tensor $\underset{\sim}{\boldsymbol{F}}={\underset{\sim}{\boldsymbol{F}}}_{i}^{e v} \boldsymbol{F}_{i}^{v}$ is decomposed into an elastic and a viscous component in the $i$ Maxwell's element, denoted respectively by the superscripts ${ }^{e v}$ and ${ }^{v}$. The arbitrary rotation of this decomposition is eliminated by fixing the viscous spin tensor ${\underset{\sim}{\boldsymbol{\Omega}} i}_{i}^{v}=\underset{\sim}{\mathbf{0}}$ [17], i.e. the viscous flow is assumed irrotational. The strain rate tensor $\underset{\sim}{\boldsymbol{D}}$ is the sum of two terms:

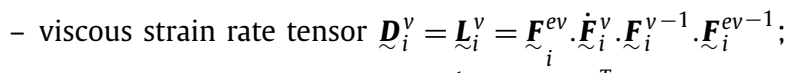

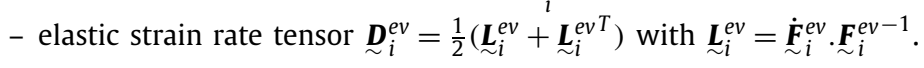




\subsection{Thermodynamics potential and state laws}

Let $\mathcal{Z}^{r m}=\left\{\underset{\sim}{\boldsymbol{C}},{\underset{\sim}{e}}_{1}^{e v}, \ldots,{\underset{\sim}{i}}_{i}^{e v}, \ldots\right\}$ be the set of internal state variables in the macromolecular network. $\underset{\sim}{\boldsymbol{C}}=\underset{\sim}{\boldsymbol{F}} . \underset{\sim}{\boldsymbol{F}}$ is the right Cauchy-Green strain tensor and $\underset{\sim}{\boldsymbol{C}}{ }_{i}^{e v}=\underset{\sim}{\boldsymbol{F}_{i}^{e v T}} \cdot{ }_{\sim}^{\boldsymbol{F}_{i}^{e v}}$ is the right Cauchy-Green tensor in the relaxed configuration. The free energy is defined as

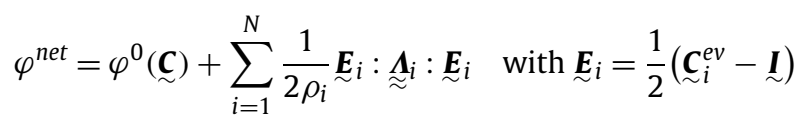

where $\rho_{i}=\rho \operatorname{det} \underset{\sim}{\boldsymbol{F}_{i}^{e v}}$ is the mass density. The network behavior is assumed to be isotropic and deviatoric for simplicity. The compressibility of the materials is assumed to be governed only by the cohesive forces in crystalline structure. In other words, the objective derivative of Cauchy stress tensors $\underset{\sim}{\widehat{\sigma}_{i}}$ is given by

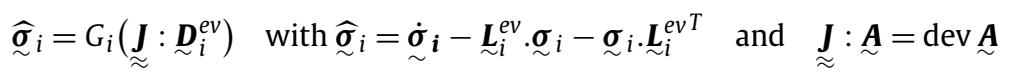

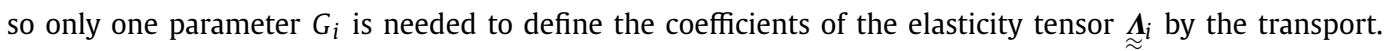

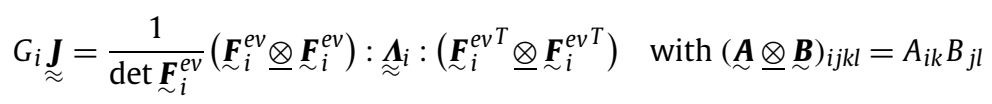

An Arruda-Boyce potential [18] defined by two scalars $(n, \mu)$ is adopted for the hyperelastic contribution of free energy $\varphi^{0}$. The state laws are derived from the free energy

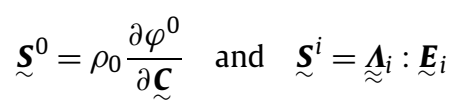

The Cauchy stress tensor in the entropic network ${\underset{\sim}{\boldsymbol{\sigma}}}^{\text {net }}$ are obtained by transport of state forces ${\underset{\sim}{\boldsymbol{S}}}^{i}$ through current configuration

$$
{\underset{\sim}{\boldsymbol{\sigma}}}^{n e t}=\frac{1}{J} \underset{\sim}{\boldsymbol{F}} \cdot{\underset{\sim}{\boldsymbol{S}}}^{0} \cdot{\underset{\sim}{\boldsymbol{F}}}^{T}+\sum_{i=1}^{n} \underset{\sim}{\boldsymbol{F}_{i}^{e v}} \cdot{\underset{\sim}{\boldsymbol{S}}}^{i} \cdot{\underset{\sim}{\boldsymbol{F}}}_{i}^{e v T} \quad \text { with }{\underset{\sim}{\boldsymbol{\sigma}}}^{i}=\underset{\sim}{\boldsymbol{F}_{i}^{e v}} \cdot{\underset{\sim}{\boldsymbol{S}}}^{i} \cdot{\underset{\sim}{\boldsymbol{F}}}_{i}^{e v T}
$$

\subsection{Evolution laws}

The dissipation potential is written as:

$$
\phi^{\text {net }}=\sum_{i=1}^{N} \frac{1}{2} \underset{\sim}{\boldsymbol{D}_{i}^{v}}: \underset{\approx}{\boldsymbol{\eta}_{i}}:{\underset{D}{i}}_{i}^{v} \quad \text { with } \underset{\approx}{\boldsymbol{\eta}_{i}}=\tau_{i} \cdot G_{i} \cdot \underset{\approx}{\boldsymbol{J}}
$$

where $\underset{\sim}{\eta_{i}}$ is fourth order viscous tensor in $i$ Maxwell element. It is defined just by the relaxation time $\tau_{i}$ and the modulus $G_{i}$. The evolution law for the Cauchy stress tensor $\underset{\sim}{\underset{\sigma}{\boldsymbol{\sigma}}}=\frac{\partial \phi^{\text {net }}}{\partial \underset{\boldsymbol{D}_{i}^{v}}{i}}$ is rewritten using Eq. (15)

$$
\widehat{\widetilde{\boldsymbol{\sigma}}}_{i}+\frac{1}{\tau_{i}} \boldsymbol{\sigma}_{i}=G_{i}(\underset{\approx}{\boldsymbol{J}}: \underset{\sim}{\boldsymbol{D}})
$$

To conclude the model part, Table 1 summarizes the current model and gives the different material parameters. A fourth order Runge-Kutta method has been used in the numerical integration using the finite element code, ZeBuLon.

\section{Uniaxial response of the model: Case of polybutene}

Two relaxation times $\tau_{1}=10 \mathrm{~s}$ and $\tau_{2}=100 \mathrm{~s}$ have been considered. Initially, the viscosity parameters $\left(G_{1}=101 \mathrm{MPa}\right.$, $G_{2}=21 \mathrm{MPa}$ ) are identified on a relaxation test before plastic deformation. The remaining material parameters $(E=$ $\left.167 \mathrm{MPa}, v=0.27, Q=28.6 \mathrm{MPa}, b=20.8, R_{0}=3.24 \mathrm{MPa}, \alpha=0.59, \beta=41.7, n=4.5, \mu=3.58 \mathrm{MPa}\right)$ are identified using monotonic and loading/unloading tensile tests. Good agreement between the experimental cyclic tensile test and numerical simulation have been obtained (Fig. 4a) with fairly good predictions of the relaxation behavior (Fig. 4b). The introduction of the damage/plasticity coupling law can account for both the slope variation at the beginning of the relaxation and the increase in the stress drop with maximal stress. 
Table 1

Visco-hyperelastic and plastic model coupled with isotropic damage.

\begin{tabular}{|c|c|c|c|}
\hline & Relevant equations & & Material parameters \\
\hline \multirow[t]{2}{*}{ Kinematic } & $\underset{\sim}{\boldsymbol{F}}={\underset{\sim}{\boldsymbol{F}^{e}} \cdot \boldsymbol{F}^{p}}^{p}$ & (Multiplicative & \\
\hline & $\underset{\sim}{\boldsymbol{F}}=\underset{\sim}{\boldsymbol{F}_{i}^{e v}} \cdot \underset{\sim}{\boldsymbol{F}_{i}^{v}}$ & decompositions) & \\
\hline Elasticity & $\underset{\sim}{\boldsymbol{\sigma}}={\underset{\sim}{\boldsymbol{\sigma}}}^{c s}+\underline{\boldsymbol{\sigma}}^{n e t}$ & (Stress partition) & $E ; \nu ; \mu ; N ; G_{i}$ \\
\hline \multirow[t]{3}{*}{ Plasticity } & ${\underset{\sim}{\nabla}}^{p}={\underset{\sim}{\dot{\boldsymbol{F}}}}^{p} \cdot{\underset{\sim}{\boldsymbol{F}}}^{p-1}=\dot{p}{\underset{\sim}{\boldsymbol{n}}}^{e}$ & (Plastic flow) & \\
\hline & $\tilde{R}=\frac{R}{1-D}=Q\left(1-\mathrm{e}^{-b \cdot p}\right)$ & (Isotropic hardening) & $b ; Q$ \\
\hline & $f \leqslant 0$ and $\dot{p} \geqslant 0$ and $\dot{p} . f=0$ & (Plastic criterion) & $R_{0}$ \\
\hline Damage & $D=\alpha\left(1-\mathrm{e}^{-\beta \cdot p}\right)$ & (Coupling law) & $\alpha ; \beta$ \\
\hline Viscosity & $\underset{\sim}{\boldsymbol{D}_{i}^{v}}=\underset{\sim}{\boldsymbol{F}_{i}^{e v}} \cdot \dot{\sim}_{i}^{v} \cdot \underbrace{\boldsymbol{F}_{i}^{v-1}}_{\sim} \cdot{\underset{\sim}{\boldsymbol{F}_{i}}}_{e v-1}^{e v}=\frac{1}{\eta_{i}} \boldsymbol{\sigma}_{i}$ & (Viscous flow) & $\tau_{i}=\frac{\eta_{i}}{G_{i}}$ \\
\hline
\end{tabular}

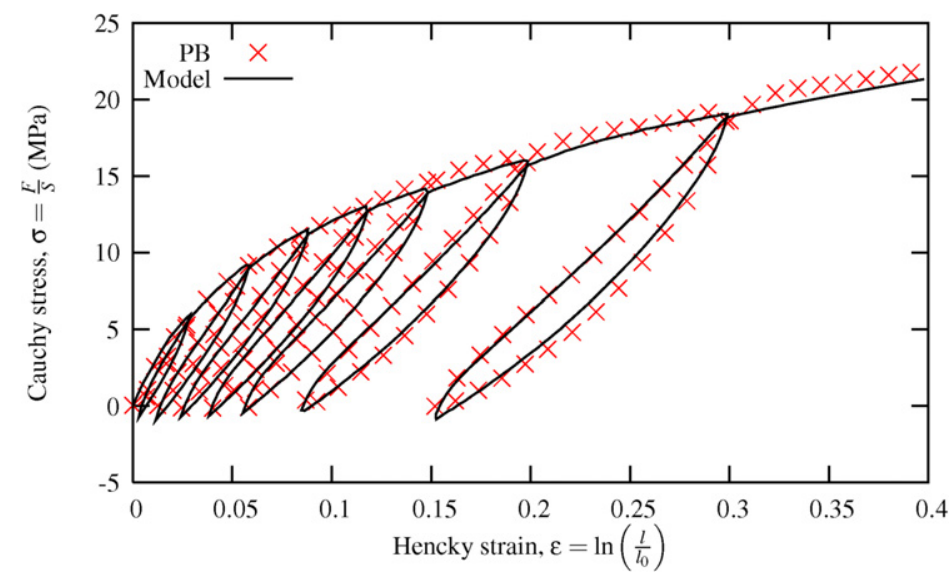

(a)

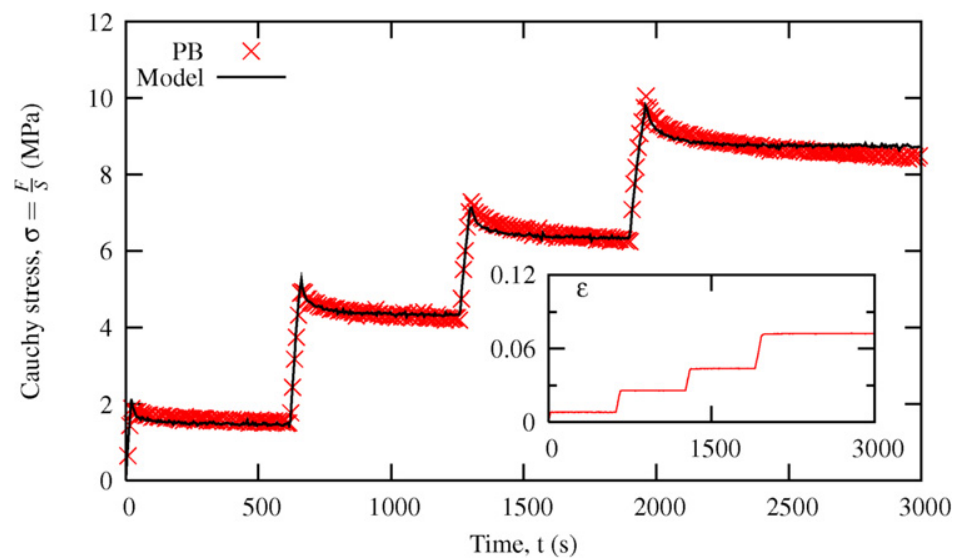

(b)

Fig. 4. (a) Load/unload tensile curve $\left(\dot{\varepsilon}=5 \times 10^{-4} \mathrm{~s}^{-1}, T=20^{\circ} \mathrm{C}\right)$. (b) Multi-relaxation curve $\left(T=20^{\circ} \mathrm{C}\right)$.

\section{Conclusion and outlook}

In this Note, the introduction of a plasticity/damage coupling has allowed us to model the loading/unloading tensile tests of semi-crystalline polymers. Moreover, relaxations behavior has been predicted by the proposed formulation.

First, an isotropic damage has been considered. However, AFM observations has revealed an anisotropic fragmentation inside the spherulites. It could be relevant to introduce a second order tensor to represent damage $\underset{\sim}{\boldsymbol{d}}$ coupled with a kinematic hardening. 


\section{Acknowledgements}

The Centre National de la Recherche Scientifique and the Région Nord/Pas-de-Calais are gratefully acknowledged for the grant of a doctoral fellowship to F. Detrez.

\section{References}

[1] K. Mrabet, R. Rahouadj, C. Cunat, An irreversible thermodynamic model for semicrystalline polymers submitted to multisequence loading at large strain, Polym. Eng. Sci. 45 (2005) 42-51.

[2] J.A.W. van Dommelen, D.M. Parks, M.C. Boyce, W.A.M. Brekelmans, F.P.T. Baaijens, Micromechanical modeling of the elasto-viscoplastic behavior of semi-crystalline polymers, J. Mech. Phys. Solids 51 (2003) 519-541.

[3] F. Bedoui, J. Diani, G. Regnier, Micromechanical modeling of elastic properties in polyolefins, Polymer 45 (2004) 2433-2442.

[4] B. Halphen, Q.S. Nguyen, Sur les matériaux standards généralisés, J. Méc. 14 (1975) 39-63.

[5] L. Laiarinandrasana, J. Besson, M. Lafarge, G. Hochstetter, Temperature dependent mechanical behavior of PVDF: Experiments and numerical modelling, Int. J. Plasticity 25 (2009) 1301-1324.

[6] P. François, V. Gaucher, R. Séguéla, Local-scale analysis of longitudinal strains in strongly necking materials by means of video-controlled extensometry, J. Phys. Condens. Matter 6 (1994) 8959-8968.

[7] G. Coulon, G. Castelin, C. G'Sell, Scanning force microscopic investigation of plasticity and damage mechanisms in polypropylene spherulites under simple shear, Polymer 40 (1999) 95-110.

[8] V. Ferreiro, G. Coulon, Shear banding in strained semicrystalline polyamide 6 films as revealed by atomic force microscopy: role of the amorphous phase, J. Polym. Sci. Polym. Phys. 42 (2004) 687-701.

[9] C. Thomas, R. Séguéla, F. Detrez, V. Miri, C. Vanmansart, Plastic deformation of spherulitic semi-crystalline polymers: An in situ AFM study of polybutene under tensile drawing, Polymer 50 (2009) 3714-3723.

[10] F. Detrez, Nanomécanismes de déformations des polymères semi-cristallins: étude in situ par microscopie à force atomique et modélisation, Thèse de l'Université de Sciences et Technologies de Lille, 2008, N ${ }^{0}$ d'ordre 4304.

[11] J. Mandel, Equations constitutives et directeurs dans les milieux plastiques et viscoplastiques, Int. J. Sol. Struct. 9 (1973) $725-740$.

[12] A. Saanouni, Sur l'analyse de la fissuration des milieux élastoviscoplastiques par la théorie de l'endommagement continu, Thèse de l'Université des Technologie de Compiègne, 1988.

[13] J. Lemaitre, A continuum damage mechanics model for ductile fracture, J. Eng. Mater. Technol. 107 (1985) 83-89.

[14] S. Cantournet, R. Desmorat, J. Besson, Mullins effect and cyclic stress softening of filled elastomers by internal sliding and friction thermodynamics model, Int. J. Sol. Struct. 46 (2009) 2255-2264.

[15] J.-L. Chaboche, Sur l'utilisation des variables d'états interne pour la description de la viscoplasticité cyclique avec endommagement, in: Problèmes Non linéaire de Mécanique, Symposium Franco-Polonais de Rhéologie et Mécanique, 1977, pp. 137-159.

[16] Q.-S. Nguyen, Stabilité et Mécanique Non Linéaire, Hermes Science, 2000.

[17] J. Sidoroff, Un modèle viscoélastique nonlinéaire avec configuration intermédiaire, J. Méc. 13 (1974) 679-713.

[18] E.M. Arruda, M.C. Boyce, A three-dimensional constitutive model for the large stretch behavior of rubber elastic materials, J. Mech. Phys. Solids 41 (1993) 389-412. 